\title{
Identification of a peptide specifically targeting ovarian cancer by the screening of a phage display peptide library
}

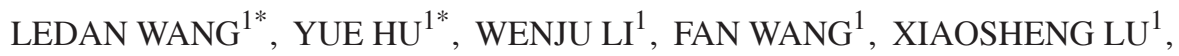 \\ XUEYING HAN ${ }^{1}$, JIEQIANG LV ${ }^{1}$ and JIE CHEN ${ }^{2}$ \\ Departments of ${ }^{1}$ Gynecology and Obstetrics and ${ }^{2}$ Children's Infectious Disease, \\ The Second Affiliated Hospital and Yuying Children's Hospital of Wenzhou Medical University, \\ Wenzhou, Zhejiang 325000, P.R. China
}

Received May 13, 2015; Accepted April 5, 2016

DOI: $10.3892 / 01.2016 .4549$

\begin{abstract}
Ovarian cancer is the most common cause of cancer-associated mortality in terms of gynecological malignancies, and is difficult to diagnose due to the absence of reliable biomarkers. To identify ovarian cancer-specific biomarkers, the present study used a Ph.D.- $7^{\text {TM }}$ Phage Display Peptide Library to screen for ligands that selectively target HO-8910 ovarian cancer cells. Following 5 rounds of biopanning, the phage clone P2 was selected by enzyme-linked immunosorbent assay and DNA sequencing, and its characteristics were additionally validated by immunofluorescence and immunohistochemical assays. The results revealed the positive phage were enriched 92-fold following 5 rounds of biopanning, and the DNA sequence AACCCGATGATTCGCCGC CAG (amino acid sequence, NPMIRRQ) was repeated most frequently (phage clones, P2, P3, P15, P30 and P54). Immunofluorescence and immunohistochemical assays revealed that the phage clone P2 was able to bind to ovarian cancer cells and tissues, and not those of cervical cancer. In conclusion, the peptide NPMIRRQ may be a potential agent for the diagnosis of ovarian cancer.
\end{abstract}

Correspondence to: Professor Jieqiang Lv, Department of Gynecology and Obstetrics, The Second Affiliated Hospital and Yuying Children's Hospital of Wenzhou Medical University, 109 Xueyuan Road, Wenzhou, Zhejiang 325000, P.R. China

E-mail: jieqianglv123@163.com

Dr Jie Chen, Department of Children's Infectious Disease, The Second Affiliated Hospital \& Yuying Children's Hospital of Wenzhou Medical University, 109 Xueyuan Road, Wenzhou, Zhejiang 325000, P.R. China

E-mail: wzyxychenjie6@163.com

${ }^{*}$ Contributed equally

Key words: ovarian cancer, phage display peptide library, HO-8910 cells, CHO cells, NPMIRRQ amino acid sequence

\section{Introduction}

Ovarian cancer is the number one cause of cancer-associated mortality out of all gynecological malignancies (1). The annual incidence of ovarian cancer is 12.7/100,000 globally, and the mortality rate is $8.1 / 100,000$ (2). In 2013, there were 22,240 women diagnosed with ovarian cancer in the United States, and 14,030 of these succumbed to disease (3). It is well-known that cancer antigen 125 (CA125) is used widely for clinical diagnosis and monitoring of the recurrence of ovarian cancer (4). However, CA125 is an unsuitable marker. Although it demonstrates elevated expression in $>80 \%$ of ovarian cancer patients, its expression is also observed in various other physiological or pathological conditions, including menstrual period, early pregnancy and endometriosis (5). Thus, the identification of specific biomarkers for early diagnosis of ovarian cancer is important and urgent.

Phage display is considered to be a powerful biological technology for screening specific peptides that bind to the surface of tumor cells (6). It was initially developed by Smith in 1985 (7). The phage-displayed random peptide libraries are first incubated on a plate coated with the target protein or cell. Then, unbound phage with no or low affinity are washed away. The eluted high-affinity phage are amplified using host bacteria and submitted to additional binding/amplification cycles to enrich the pool in favor of binding sequences. After 3-5 rounds, individual clones are characterized by DNA sequencing and enzyme-linked immunosorbent assay (ELISA) (8) to obtain the corresponding structural and functional information.

The present study aimed to identify optimal biomarkers for ovarian cancer by using a Phage Display Peptide Library to screen for ligands that selectively target ovarian cancer cells. The results of the present study may assist with the diagnosis of ovarian cancer and the phages identified may be utilized as carriers for drug delivery.

\section{Materials and methods}

Cells and reagents. The HO-8910 human ovarian cancer cell line, and HeLa cervical cancer cell line, were purchased from Jilin Baili Biotechnology Co., Ltd. (Changchun, China). The 
Chinese hamster ovary cell line (CHO) was purchased from Sangon Biotech Co., Ltd. (Shanghai, China). Dulbecco's modified Eagle's medium (DMEM) and RPMI-1640 medium were purchased from Sangon Biotech Co., Ltd. HeLa cells were cultured in RPMI-1640, while the other cells were cultured in DMEM supplemented with $10 \%(\mathrm{v} / \mathrm{v})$ fetal bovine serum, at $37^{\circ} \mathrm{C}$ with $5 \% \mathrm{CO}_{2}$.

Escherichia coli ER2738 was purchased from Biovector Co.,Ltd. (Beijing, China). The M13K07 phage, anti-M13 mouse monoclonal antibody (\#27-9421-01), horseradish peroxidase (HRP)-conjugated goat anti-mouse antibody (\#sc-2005) and fluorescein isothiocyanate (FITC)-labeled goat anti-mouse antibody (\#sc-2010) were purchased from Sangon Biotech Co., Ltd..

Phage display biopanning procedures. The Ph.D.-7 Phage Display Peptide Library kit was purchased from New England BioLabs, Inc. (Ipswich, MA, USA). Screening procedures were performed according to the manufacturer's protocol (version 1.0) with some modifications. Firstly, CHO and HO-8910 cells were digested with trypsin and the number of cells was adjusted to $1 \times 10^{7} / \mathrm{ml}$. Subsequently, $100 \mu 1 \mathrm{CHO}$ cells were transferred to an Eppendorf tube and $10 \mu \mathrm{l}$ of the Ph.D.-7 Phage-Display Peptide Library was added, which initially contained $2 \times 10^{11}$ plaque-forming units (pfu). The cells were incubated at $4^{\circ} \mathrm{C}$ for $2 \mathrm{~h}$. A total of $200 \mu \mathrm{l}$ organic solvent was added to the tube, which consisted of $180 \mu \mathrm{l}$ dibutyl phthalate (DBP) and $20 \mu \mathrm{l}$ cyclohexane (Beijing Yiqiangsheng Technology Co., Ltd., Beijing China). The tube was subsequently centrifuged at $10,000 \times \mathrm{g}$ for $10 \mathrm{~min}$. Following centrifugation, the soluble fluid upper layer was pipetted into a fresh tube, which contained HO-8910 cells, and was incubated at $4^{\circ} \mathrm{C}$ for $3 \mathrm{~h}$. The precipitate was transferred to a fresh tube, and $200 \mu \mathrm{l}$ Luria-Bertani (LB) with E. coli ER2738 (mid-log phase) was added and incubated at $37^{\circ} \mathrm{C}$ for $30 \mathrm{~min}$. Subsequently, phage was titrated and amplified, according to the manufacturer's instructions (New England BioLabs, Inc.; www.neb.com/protocols/2014/ 05/08/m13-titer-protocol). Finally, 5 rounds of in vitro reiterative biopanning were performed.

Selection and amplification of positive clones. Following 5 rounds of biopanning, 60 blue plaques were randomly selected, and were individually added to E. coli ER2738 cultures for amplification and titration.

ELISA. HO-8910 cells were plated into 96-well plates at a density of $10^{4}$ cells/well. Following $1 \mathrm{~h}$ of incubation at $37^{\circ} \mathrm{C}$, the selected positive phage clones $\left(10^{10} \mathrm{pfu} /\right.$ well $), \mathrm{M} 13 \mathrm{~K} 07$ phage and phosphate-buffered saline (PBS), were added individually to the cells and incubated at $37^{\circ} \mathrm{C}$ for $2 \mathrm{~h}$. Subsequently, the cells were washed three times with PBS and cultured at $37^{\circ} \mathrm{C}$ for $2 \mathrm{~h}$ in the presence of anti-M13 mouse monoclonal antibody (dilution, 1:6,000). Subsequently, the plates were washed and HRP-conjugated goat anti-mouse antibody (dilution, 1:4,000) was added. Following $2 \mathrm{~h}$ of incubation, the plates were washed and $200 \mu \mathrm{l}$ fresh substrate solution (3,3',5,5'-tetramethylbenzidine; Sigma-Aldrich China, Inc., Shanghai, China) was added to each well, and the absorbance values at $450 \mathrm{~nm}$ were recorded using a plate reader.
Table I. Biopanning of the HO-8910 ovarian cancer cell line using a phage display peptide library.

\begin{tabular}{lccc}
\hline Round & $\begin{array}{c}\text { Input } \\
\text { number, pfu }\end{array}$ & $\begin{array}{c}\text { Output } \\
\text { number, pfu }\end{array}$ & $\begin{array}{c}\text { Recovery } \\
\text { rate }^{\mathrm{a}}\end{array}$ \\
\hline 1 & $2.0 \times 10^{11}$ & $5.0 \times 10^{5}$ & $2.5 \times 10^{-6}$ \\
2 & $1.5 \times 10^{11}$ & $1.0 \times 10^{6}$ & $6.7 \times 10^{-6}$ \\
3 & $1.8 \times 10^{11}$ & $3.0 \times 10^{6}$ & $1.7 \times 10^{-5}$ \\
4 & $2.5 \times 10^{11}$ & $1.0 \times 10^{7}$ & $4.0 \times 10^{-5}$ \\
5 & $1.6 \times 10^{11}$ & $3.6 \times 10^{7}$ & $2.3 \times 10^{-4}$ \\
\hline
\end{tabular}

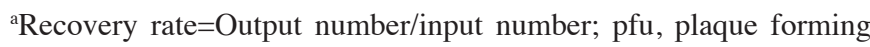
unit.

DNA sequencing. A total of 13 phage clones were selected if their optical density (OD) ${ }_{450}$ was $>0.6$, and the single stranded DNA from the positive phages was purified using an M13 purification kit (Beijing Sunny Instruments Co., Ltd., Beijing, China) according to the manufacturer's protocol. The samples were sent to Sangon Biotech Co., Ltd. for sequencing, and the sequences were analyzed by using Vector NTI Advance ${ }^{\circledR}$ software (version 10.3; Thermo Fisher Scientific, Inc., Waltham, MA, USA).

Immunofluorescence assay. HO-8910, HeLa and CHO cells were seeded onto glass coverslips at $2 \times 10^{4} / \mathrm{ml}$, and grown to $80 \%$ confluence. Cells were washed gently with PBS, and fixed in $4 \%$ paraformaldehyde for $15 \mathrm{~min}$ at room temperature. The selected targeting phage clone P2 and PBS were added individually to the cells and incubated at $37^{\circ} \mathrm{C}$ for $2 \mathrm{~h}$, following by washing with PBS three times. Cells were subsequently incubated with anti-M13 mouse monoclonal antibody (dilution, 1:500). Following $1 \mathrm{~h}$ of incubation at $37^{\circ} \mathrm{C}$, the cells were washed 10 times with PBST, and FITC-labeled goat anti-mouse antibody (dilution, 1:500) and propidium iodide (dilution, 1:1,000) were added. Following incubation at $37^{\circ} \mathrm{C}$ for $30 \mathrm{~min}$, the cells were visualized using a fluorescence microscope.

Immunohistochemical staining. The selected phage clone was added to ovarian cancer and normal ovarian tissue samples, which were obtained from the Department of Gynecology and Obstetrics, The Second Affiliated Hospital \& Yuying Children's Hospital of Wenzhou Medical University (Wenzhou, China). Following $30 \mathrm{~min}$ of incubation at room temperature, the tissue samples were sequentially incubated with anti-M13 mouse monoclonal antibody (dilution, 1:300) for $1 \mathrm{~h}$, followed by incubation with the HRP-conjugated goat anti-mouse antibody (dilution, 1:100). A total of $1 \mathrm{~h}$ later, 3,3'-diaminobenzidine (DAB) solution was added and the samples were visualized using a light microscope.

\section{Results}

Phage display biopanning. In the present study, a 7-mer phage display library was employed to screen the peptides binding specifically to the HO-8910 ovarian cancer cell line. The 
Table II. DNA sequences of the 13 clones.

\begin{tabular}{|c|c|c|c|}
\hline Clone & DNA sequence & Amino acid sequence & Frequency \\
\hline P2, P3, P15, P30, P54 & AACCCGATGATTCGCCGCCAG & NPMIRRQ & 5 \\
\hline P9, P35, P58 & ATGCGCATGACCATTATTAAC & MRMTIIN & 3 \\
\hline P57 & CTGCGCCTGCGCAACACCCGC & LRLRNTR & 1 \\
\hline P12 & AGCCATCTGCGCCATCGCATT & SHLRHRI & 1 \\
\hline P37 & AAACTGCCGCTGACCACCAAA & KLPLTTK & 1 \\
\hline P60 & CCGATTAAAACCAACCGCAAA & PIKTNRK & 1 \\
\hline P48 & AAACCGACCATTCCGACCAAA & KPTIPTK & 1 \\
\hline
\end{tabular}

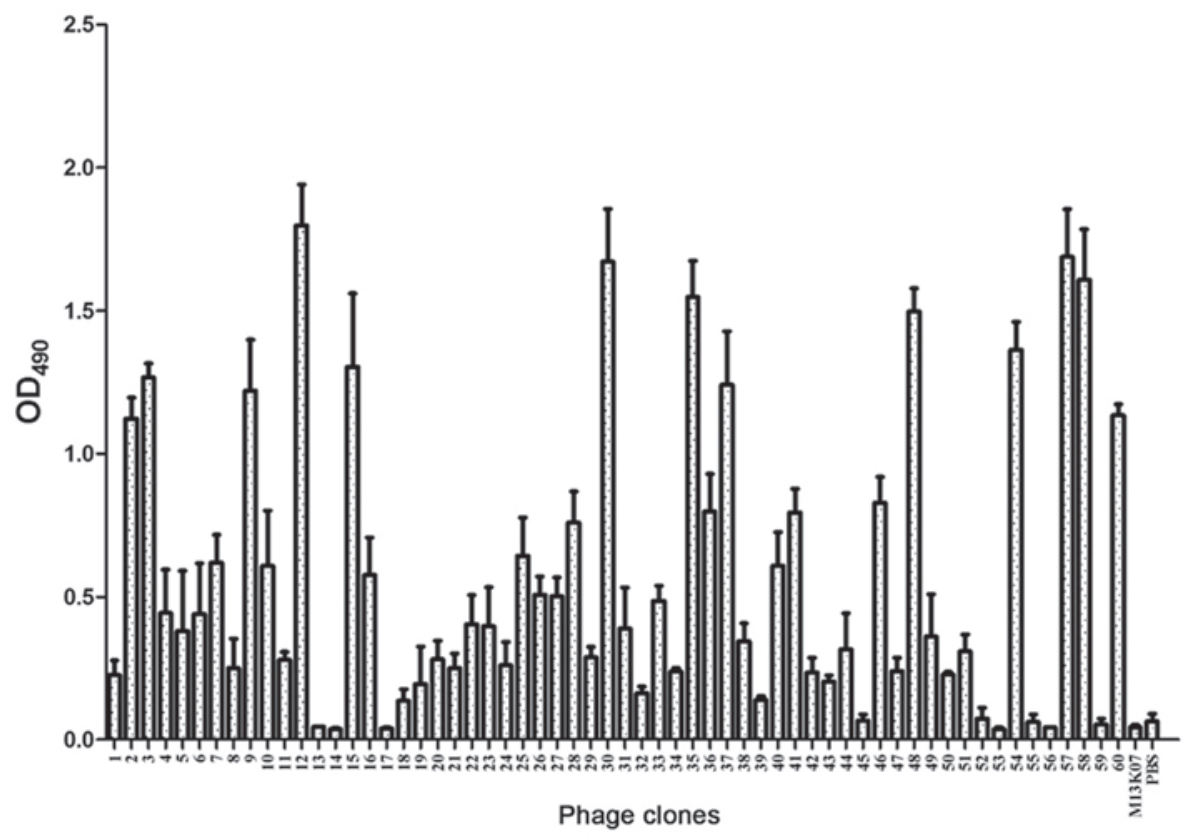

Figure 1. Enzyme-linked immunosorbent assay (ELISA) of the selected phage clones binding to HO-8910 cells. After 5 rounds of biopanning, 60 plaques were randomly selected and analyzed using ELISA. The $\mathrm{OD}_{490}$ value of 13 clones was $>0.6$ (P2, P3, P9, P12, P15, P30, P35, P37, P48, P54, P57, P58 and P60). The M13K07 phage and phosphate-buffered saline were used as negative controls. OD, optical density.

results of this screening (Table I) revealed that the recovery rate increased 92-fold following 5 rounds of biopanning, which demonstrated that the phages binding to HO-8910 cells were enriched.

ELISA and DNA sequencing. Following 5 rounds of biopanning, the individual phages were randomly selected and analyzed using ELISA assay. As demonstrated in Fig. 1, the $\mathrm{OD}_{490}$ value of 13 clones was $>0.6$, and these 13 phage clones (P2, P3, P9, P12, P15, P30, P35, P37, P48, P54, P57, P58 and P60) were sequenced. As demonstrated in Table II, 7 distinct sequences from the 13 clones were obtained and the most frequent DNA sequence was AACCCGATGATTCGCCGC CAG; the corresponding amino acid sequence was NPMIRRQ.

Immunocytochemical staining and immunofluorescence assay. According to the results of the phage clones DNA sequence analysis, the most frequent sequence (NPMIRRQ) was selected, and as P2, P3, P15, P30 and P54 all contained the same amino acid sequence, the corresponding phage clone
P2 was selected to perform immunocytochemical staining and immunofluorescence assay to additionally confirm the specificity of the phage clone binding to HO-8910 cells. As demonstrated in Fig. 2, a fluorescent signal was observed in the HO-8910 cells, and no fluorescent signal was observed in the HeLa and CHO cells.

Immunohistochemical staining results. In Fig. 3, positive DAB staining (brown color) was observed in the ovarian cancer tissue incubated with phage clone $\mathrm{P} 2$, and not observed in normal ovarian tissue.

\section{Discussion}

In recent years, tumor-targeting therapy has become one of the focuses of clinical cancer treatment, with the aim of using highly-specific tumor material as a carrier, which will be able to specifically deliver anti-tumor drugs to the tumor site (9). Small molecule peptides may be an ideal carrier of anti-tumor targeted drugs, due to their simple 

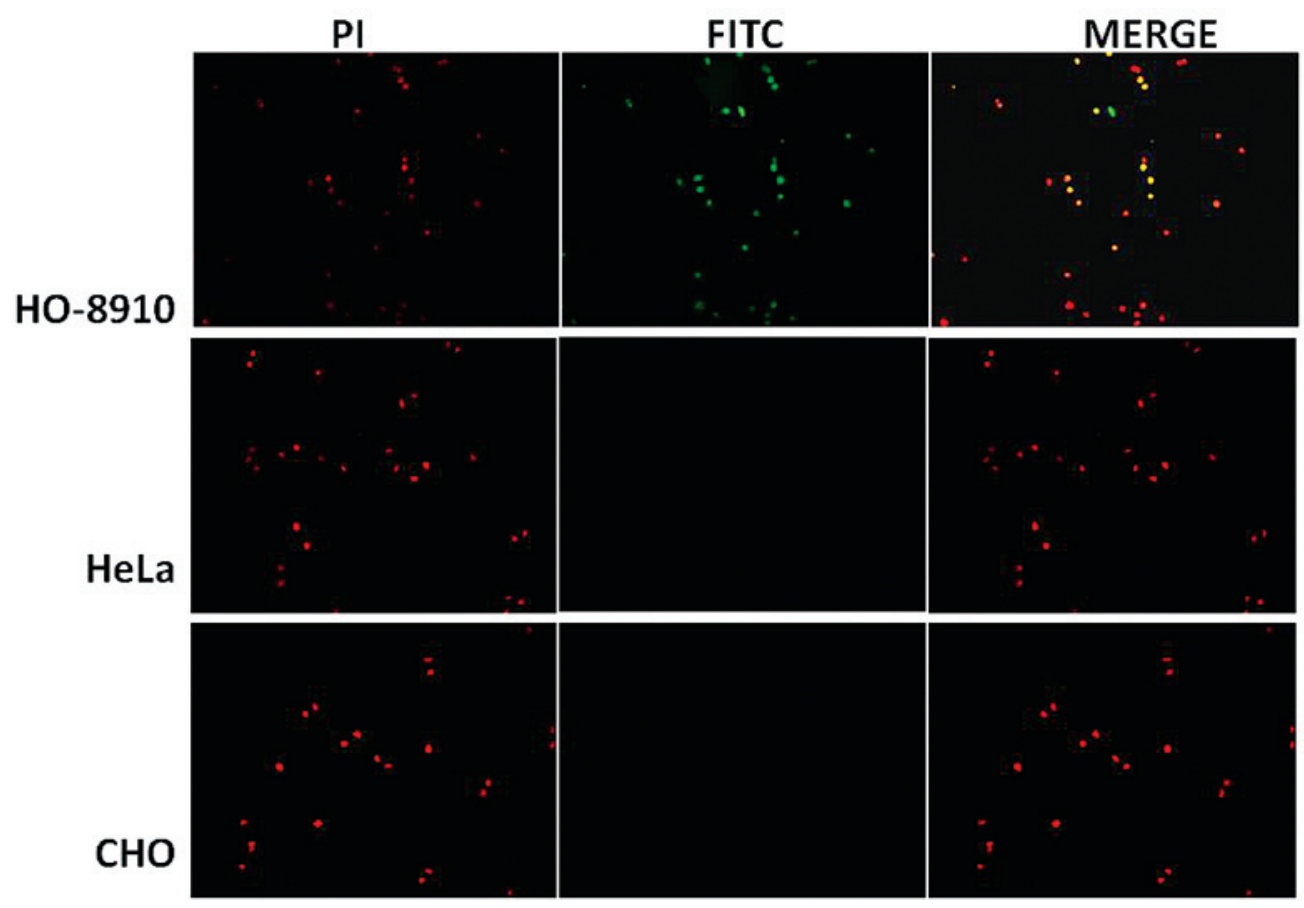

Figure 2. Immunofluorescence assay of phage clone P2 binding HO-8910, HeLa and CHO cells. The selected phage clone P2 was added individually to the cells and then incubated with anti-M13 antibody, FITC-labeled antibody and PI. The results were visualized using a fluorescence microscope. The results show that P2 could bind to HO-8910 cells but not to HeLa and CHO cells (control cells). Magnification, x200. PI, propidium iodide; FITC, fluorescein isothiocyanate.

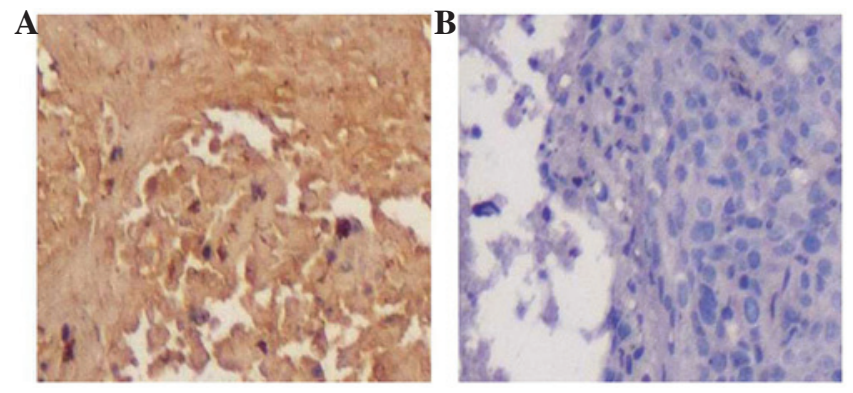

Figure 3. 3,3'-diaminobenzidine (DAB) staining of phage clone $\mathrm{P} 2$ incubated with (A) ovarian cancer and (B) normal ovarian tissue. The P2 clone was added to ovarian cancer and normal ovarian tissue samples, incubated with anti-M13 antibody, horseradish peroxidase-conjugated antibody and DAB solution, then visualized using a light microscope. The results show that $\mathrm{P} 2$ could bind to ovarian cancer tissue (brown staining) but not normal ovarian tissue. Magnification, x100.

structure, easy preparation and strong penetration into the tumor tissue (10). Phage display technology is able to rapidly screen high-affinity antibodies or peptide ligands that bind with specific target molecules (11). The advantage of this technology is that it realizes the linkage between genotype and phenotype (12), and there is no need to know the structural information of the target molecule in advance (13). It is possible to obtain the amino acid sequence indirectly by sequencing the positive phage clones following a screening procedure.

Recently, peptides targeting different tumor cells have been identified using phage display technology $(14,15)$. For the ovarian cancer cell line SKOV3, the peptides WSGPGVWGASVK (16) and SVSVGMKPSPRP (17) were identified to have high affinity. However, there are various pathological types of ovarian cancer, and there is a limited amount of research focusing on the targeting of these peptides against the ovarian cancer HO-8910 cell line. In the present study, a 7-mer phage display peptide library was used to isolate specific peptides binding specifically to HO-8910 cells. After 5 rounds of biopanning, the recovery rate of phages demonstrated a 92-fold increase over the first round, indicating that the bound phage were amplified. Subsequently, blue plaques were randomly selected after 5 rounds of biopanning. According to the results of DNA sequencing, the 13 phages contained 7 distinct polypeptide sequences, and the amino acid sequence NPMIRRQ was enriched significantly.

To additionally identify the affinity of the positive clones, the phage clone P2 was selected for further study. Immunocytochemical staining and immunofluorescence assay were performed to confirm the specificity of the phage clone P2 binding to HO-8910 cells. The results of immunocytochemical staining and immunofluorescence assay suggested that the phage clone P2 was able to bind to HO-8910 cells, and not the HeLa cervical cancer cell line.

In conclusion, the present study identified a novel peptide, NPMIRRQ, targeting ovarian cancer was isolated using phage display. The peptide was able to bind to target cells and ovarian cancer tissues specifically, and therefore potentially be applied in the diagnostics and treatment of ovarian cancer.

\section{Acknowledgements}

The present study was supported by grants from the Zhejiang Provincal Natural Science Foundation (no. LQ16H160022), the Zhejiang Provincal Technology Bureau, Zhejiang, China (no. 2014C37003) and Wenzhou Technology Bureau, Wenzhou, China (no. 2015Y0372). 


\section{References}

1. Rooth C: Ovarian cancer: Risk factors, treatment and management. Br J Nurs 22: S23-S30, 2013.

2. Collins Y, Holcomb K, Chapman-Davis E, Khabele D and Farley JH: Gynecologic cancer disparities: A report from the Health Disparities Taskforce of the Society of Gynecologic Oncology. Gynecol Oncol 133: 353-361, 2014.

3. Siegel R, Naishadham D and Jemal A: Cancer statistics, 2013. CA Cancer J Clin 63: 11-30, 2013.

4. Bocheva Y, Bochev P and Ivanov S: Ca-125 in diagnosis and monitoring of patients with ovarian cancer. Akush Ginekol (Sofiia) 54: 11-7, 2015

5. CannistraSA:Cancerof the ovary.NEnglJMed351:2519-2529,2004.

6. Jemal A, Siegel R, Xu J and Ward E: Cancer statistics, 2010. CA Cancer J Clin 60: 277-300, 2010.

7. Smith GP: Filamentous fusion phage: Novel expression vectors that display cloned antigens on the virion surface. Science 228 : $1315-1317,1985$

8. Parmley SF and Smith GP: Antibody-selectable filamentous fd phage vectors: Affinity purification of target genes. Gene 73: 305-318, 1988.

9. Velasco-Velázquez M, Xolalpa W and Pestell RG: The potential to target CCL5/CCR5 in breast cancer. Expert Opin Ther Targets 18: 1265-1275, 2014

10. Bastien JI, McNeill KA and Fine HA: Molecular characterizations of glioblastoma, targeted therapy, and clinical results to date. Cancer 121: 502-516, 2015.
11. Henry KA, Arbabi-Ghahroudi $M$ and Scott JK: Beyond phage display: Non-traditional applications of the filamentous bacteriophage as a vaccine carrier, therapeutic biologic, and bioconjugation scaffold. Front Microbiol 6:755, 2015

12. Kaplan G and Gershoni JM: A general insert label for peptide display on chimeric filamentous bacteriophages. Anal Biochem 420: 68-72, 2012.

13. Silacci M, Brack S, Schirru G, Mårlind J, Ettorre A, Merlo A, Viti $F$ and Neri D: Design, construction, and characterization of a large synthetic human antibody phage display library. Proteomics 5: 2340-2350, 2005.

14. Lee KJ, Lee JH, Chung HK, Ju EJ, Song SY, Jeong SY and Choi EK: Application of peptide displaying phage as a novel diagnostic probe for human lung adenocarcinoma. Amino Acids 48: 1079-1086, 2016.

15. Che YJ, Wu HW, Hung LY, Liu CA, Chang HY, Wang K and Lee GB: An integrated microfluidic system for screening of phage-displayed peptides specific to colon cancer cells and colon cancer stem cells. Biomicrofluidics 9: 054121, 2015.

16. Ma C1, Yin G, Yan D, He X, Zhang L, Wei Y and Huang Z: A novel peptide specifically targeting ovarian cancer identified by in vivo phage display. J Pept Sci 19: 730-736, 2013.

17. Zhang L, Yin G, Yan D, Wei Y, Ma C, Huang Z, Liao X, Yao Y, Chen $\mathrm{X}$ and Hao B: In vitro screening of ovarian tumor specific peptides from a phage display peptide library. Biotechnol Lett 33: $1729-1735,2011$. 